\title{
Vibrational convection in a heterogeneous binary mixture. Part I. Time-averaged equations
}

\author{
Anatoliy Vorobev $^{1} \dagger$ and Tatyana Lyubimova ${ }^{2,3}$ \\ ${ }^{1}$ Faculty of Engineering and Physical Sciences, University of Southampton, SO17 1BJ, UK \\ ${ }^{2}$ Institute of Continuous Media Mechanics Ural Branch RAS, Perm, Russia \\ ${ }^{3}$ Perm State University, Perm, Russia
}

(Received $\mathrm{xx}$; revised $\mathrm{xx}$; accepted $\mathrm{xx}$ )

High-frequency vibrations of a container filled with a fluid generate the pulsation flows that however are barely visible with the naked eye, and induce the slow but largeamplitude averaged flows that are important for various practical applications. In this work we derive a theoretical model that gives the averaged description of the influence of uniform high-frequency vibrations on an isothermal mixture of two slowly miscible liquids. The miscible multiphase system is described within the framework of the phasefield approach. The full Cahn-Hillard-Navier-Stokes equations are split into the separate systems for the quasi-acoustic, pulsating and averaged flow fields, eliminating the need for the resolution of the short time-scale pulsation motion and thus making the analysis of the long-term evolution much more efficient. The resultant averaged model includes the effects of concentration diffusion and barodiffusion, the dynamic interfacial stresses, and the generation of the hydrodynamic flows by non-homogeneities of the concentration field (when they are combined with the effects of gravity and vibrations). The resultant model for the vibrational convection in a heterogeneous mixture of two fluids separated by diffusive boundaries could be used for the description of processes of mixing/de-mixing, solidification/melting, polymerisation, etc. in the presence of vibrations.

\section{Introduction}

Forced vibrations can be used to control the heat/mass transfer in fluid systems, the position of interfaces, the buoyancy conditions for solid or fluid inclusions, etc. High-frequency vibrations of a container filled with a fluid induce the small-amplitude pulsation motion, that in most cases is hardly-visible with the naked eye, and the slow but large-amplitude averaged motion that is much more important for practical applications (e.g. microfluidics, control of crystal growth from melt, and other chemical engineering processes) (see Gershuni \& Lyubimov 1998; Lyubimov et al. 2003).

The effective description of the high-frequency vibrations is achieved with the use of the multiple-scale method and the averaging procedure, which could be utilised for splitting the physical processes that occur on different time scales, thus representing the hydrodynamic evolution as the small-amplitude pulsations on the background of slower large-amplitude changes, and thus splitting the governing equations for the separate, although coupled, sets of pulsation and averaged equations. The use of the averaged equations allows the time integration with much larger time steps as the need to resolve the short-scale oscillatory motion is eliminated. In addition, the high-frequency

$\dagger$ Email address for correspondence: A.Vorobev@soton.ac.uk 
vibrations are known to generate thin skin-layers near rigid boundaries and interfaces (see Schlichting \& Gersten 2017; Landau \& Lifshitz 1987). The multiple-scale analysis of flow fields in these boundary layers allows the derivation of the so-called effective boundary conditions that give the values of the variables at the outer side of the skin-layer making the numerical resolution of the thin skin-layers unnecessary, and thus enormously reducing the computational time required for the numerical solution of similar problems.

In the current work, we develop a theoretical model for a heterogeneous (with interfaces) binary mixture of two slowly miscible liquids that are enclosed in a container that is subjected to the high-frequency small-amplitude mechanical vibrations. The traditional description of the multiphase system is given within the Laplacian approach, when the phases are divided by infinitely thin boundaries endowed with the surface tension (see e.g. Landau \& Lifshitz 1987). To describe the hydrodynamic processes in the multiphase system, the governing equations are separately solved within each phase, and then the obtained solutions are matched by using the appropriate boundary conditions imposed at the interfaces. The traditional description becomes inconvenient if the interfaces experience strong topological transformations, or may even emerge or disappear during the evolution of the binary system (which may occur e.g. due to dissolution of existent inclusions or due to nucleation of inclusions of a new phase).

An alternative description is to represent the interface as a transition layer of a finite thickness across which almost all variables experience sharp but still continuous changes. One set of the governing equations is solved for the whole multiphase system including the interface. The position of the interface is determined on the basis of the so-called phase function. The governing equations also include the new terms that take into account the surface tension effects. This continuum approach is primarily used for the numerical description of the complex evolution of multiphase systems, and this approach is practically realised by three alternative techniques: the volume-of-fluid, the level set, and the phase-field methods. These techniques are quite similar, although the two former approaches are formulated on the bases of the mathematical ideas developed for the description and tracking of the curved interfaces, and the phase-field approach is based on the physical background, namely, the consistent thermodynamic model for the binary mixture of two miscible fluids (see Vorobev 2014).

The effects of vibrations on the behaviour of a multiphase system were earlier described within the framework of the traditional (Laplacian) approach, and these results are summarised in books by Gershuni \& Lyubimov (1998) and Lyubimov et al. (2003). It was shown that the high-frequency vibrations excite the average flows in the bulk, that is described by the additional vibrational force in the Navier-Stokes equation, and the vibrations can also generate the average motion near the rigid walls or interfaces, and this motion can be efficiently captured by the use of the effective boundary conditions (this motion is frequently termed as the acoustic streaming (see e.g. Mason 1965; Landau \& Lifshitz 1987)). In the current work, we develop the phase-field model to describe the effects of the high-frequency vibrations on the heterogeneous mixture of two slowly miscible liquids.

Dynamics of miscible systems under the influence of high-frequency vibrations was previously studied in a number of experimental and numerical works (see Legendre et al. 2003; Gaponenko et al. 2006; Gaponenko \& Shevtsova 2010; Gaponenko et al. 2015a,b; Shevtsova et al. 2015, 2016; Wolf 2018). The theoretical description that was adopted in all previous papers was based on the traditional approach that presumes that a mixture of two liquids that are miscible in all proportions can be represented by a single-phase model, i.e. as a homogeneous binary mixture with the mixture components distinguished by the different levels of concentration. This description is mathematically equivalent 
to the problem of thermal vibrational convection, when the equation for the balance of species is replaced by the equation for the temperature field (e.g. the thermal vibrational convection of fluids near the critical point was studied by Lyubimov et al. 2006a,b; Zappoli et al. 2015).

This classical approach however completely disregards interfacial stresses that exist at a liquid/liquid boundary. Indeed, an everyday observation of dissolution of honey droplet in tea points out that the concept of an interface endowed with surface tension is required for an accurate description of such a process, as one can observe a sharp clearly visible boundary that separates two liquids for a long time period, and one can also see that the honey droplet tends to be of a spherical shape (see Joseph \& Renardy 1993; Vorobev 2014). In our current work we develop a theoretical model that takes into account the dynamic surface tension associated with miscible interfaces.

\section{Phase-field approach}

We consider a binary mixture that occupies a closed container. The container is subjected to the high-frequency uniform translational vibrational forcing determined by the pulsation velocity $\boldsymbol{w}_{0}=a \omega \boldsymbol{j} \cos (\omega t)$, where $a$ and $\omega$ are the amplitude and angular frequency of the vibrations, and the unit vector $j$ determines the direction of the vibrations. $\dagger$ We call these vibrations uniform, to underline that this is the spatially uniform vibrational forcing, i.e. both $a$ and $\boldsymbol{j}$ are independent of the spacial coordinates: all walls of a container oscillate with the same amplitude and in the same direction. The non-uniform (e.g. rotational) vibrations may induce some different effects, and the derivation of the averaged equations for such vibrations would be also different (for more details see Gershuni \& Lyubimov 1998).

The vibrations can be included into the hydrodynamic model by adding the pulsation velocity to the velocity boundary conditions imposed at the container's walls. This approach presumes that the problem is solved by using the immovable laboratory reference frame. A more convenient approach is to change the reference frame and to solve the problem by using the reference frame that is attached to the vibrating container. The new reference frame is non-inertial, and thus a new inertial (fictitious) force, $-\rho \frac{\partial \boldsymbol{w}_{0}}{\partial t}$, is to be added to the momentum-balance equation.

The full set of the Navier-Stokes-Cahn-Hilliard equations that determines the thermoand hydrodynamic evolution of an isothermal heterogeneous binary mixture were first obtained by Lowengrub \& Truskinovsky (1998). These equations include the laws for the conservation of mass, species, and momentum (which we augment by adding the inertial force),

$$
\begin{gathered}
\frac{\partial \rho}{\partial t}+\nabla \cdot(\rho \boldsymbol{v})=0 \\
\rho\left(\frac{\partial C}{\partial t}+(\boldsymbol{v} \cdot \nabla) C\right)=\alpha \nabla^{2} \mu \\
\rho\left(\frac{\partial \boldsymbol{v}}{\partial t}+(\boldsymbol{v} \cdot \nabla) \boldsymbol{v}\right)=-\nabla p+\nabla \cdot \tau_{\eta}-\epsilon \nabla \cdot \tau_{\epsilon}+\rho \boldsymbol{g}-\rho \frac{\partial \boldsymbol{w}_{0}}{\partial t} .
\end{gathered}
$$

Here the conventional notations are accepted for all variables, namely, $t$ is the time, $\boldsymbol{v}$ is the velocity vector (that is defined as the mass-averaged velocity of fluid particle

$\dagger$ The pulsation velocity can be determined by an arbitrary periodic function of time. Expansion of a periodic function into a Fourier series would reduce the description of any periodic function to the one discussed in this work. 
composed of two different liquids), $\rho$ is the density, $p$ is the pressure, $C$ is the concentration (defined as the mass fraction of one of the components in the mixture), $\mu$ is the chemical potential, and $\boldsymbol{g}$ is the gravity acceleration.

To take into account the surface tension effect, the equation for the momentum balance includes an additional force that is called the Korteweg force (see e.g. Joseph \& Renardy 1993). The magnitude of this term is determined by the parameter $\epsilon$ called the capillary constant.

The viscous and Korteweg stress tensors in the equation for the momentum balance (2.3) are defined by the following expressions:

$$
\begin{gathered}
\tau_{\eta}=\eta\left(\nabla \otimes \boldsymbol{v}+(\nabla \otimes \boldsymbol{v})^{T}-\frac{2}{3}(\nabla \cdot \boldsymbol{v}) I\right), \\
\tau_{\epsilon}=\rho \nabla C \otimes \nabla C .
\end{gathered}
$$

Here the superscript $T$ means the transpose of a matrix, and $I$ stands for the unit matrix. The coefficient of viscosity $\eta$ is in general a function of the mixture density.

The diffusive term in the equation for the species balance (2.2) is determined on the basis of the extended Fick's law, i.e. with the diffusion flux defined by the gradient of the chemical potential (Landau \& Lifshitz 1987). The mobility coefficient $\alpha$ can be related to the coefficient of diffusion as $D=\frac{\alpha}{\rho}\left(\frac{\partial \mu}{\partial C}\right)_{p}$.

For the equation of state, we use the relation for 'simple mixtures' that assumes that there is no volume gain or loss upon mixing of two substances,

$$
\frac{1}{\rho}=\frac{1-C}{\rho_{01}}+\frac{C}{\rho_{02}},
$$

where $\rho_{01}$ and $\rho_{02}$ are the densities of the pure components.

Finally, the chemical potential is defined by the expression (see Lowengrub \& Truskinovsky 1998):

$$
\mu=\mu_{0}(C)-\frac{p}{\rho^{2}} \frac{d \rho}{d C}-\frac{\epsilon}{\rho} \nabla \cdot(\rho \nabla C),
$$

where $\mu_{0}$ is the classical part of the chemical potential that is determined from the free energy function $\mu_{0}=\frac{d f_{0}}{d C}$.

The formulated mathematical model needs to be completed with the set of the boundary conditions,

$$
\boldsymbol{v}=0, \frac{\partial \mu}{\partial n}=0, \frac{\partial C}{\partial n}=0 .
$$

The first condition is the standard no-slip boundary condition. The second condition states the zero diffusive flux through the rigid walls. Here $\boldsymbol{n}$ is the unit vector normal to the wall, and thus $\partial /(\partial n)$ denotes the normal derivative.

Since the set of the governing equations is of the higher, forth, order in terms of the concentration field the additional condition for the concentration field is needed. This condition mimics the wetting properties of the wall and liquid/liquid pair, setting the relative strengths of the interactions of the molecules of the mixture components with the wall. Here we impose one of the simplest forms of this conditions, which implies that the wall interacts neutrally with the mixture components, and thus the contact line is orthogonal to the wall.

We would like to make two additional comments regarding equations (2.1)-(2.3). First, we limit our analysis to the consideration of the evolution of an isothermal binary system, assuming that there are no externally imposed temperature gradients and also neglecting the effects of internal generation of the temperature inhomogeneities (see Umantsev 
2002). In general, phase transitions always occur with the release or absorption of some latent heats (Landau \& Lifshitz 1987). For some processes, e.g. solidification/melting, the release or absorption of the latent heat determines the propagation of the phase boundaries. We however focus on the problems of mixing/de-mixing, assuming that the phase separation is solely driven by the concentration differences, and that the enthalpy of phase separation is relatively small (for instance, for the binary mixture of polymers in water studied in Gan et al. (2000), the enthalpy of phase separation was reported to be $21 \mathrm{~J} / \mathrm{g}$, which is considerably smaller than e.g. the heat of vaporisation of water, $2.3 \mathrm{~kJ} / \mathrm{g}$, or the latent heat of ice melting, $330 \mathrm{~J} / \mathrm{g}$ ), so that this amount of heat can be rapidly diffused to the interface (or away from the interface) permitting us to assume that the mixing/de-mixing processes occur under isothermal conditions. No temperature differences are reported in the recent experiments (Gaponenko et al. 2015a,b) where the influence of vibrations on dynamics of two miscible liquids (water/isopropanol) was studied.

Second, we consider the evolution of two incompressible liquids, i.e. assuming that the mixture density is independent of pressure. Nevertheless, owing to the dependence of the mixture density on concentration the full continuity equation still needs to be used (which is equation (2.1)). This effect is called the quasi-compressibility and the hydrodynamic processes that are associated with this effect are called the quasi-acoustics. The necessity to solve the full continuity equation is the major difficulty in the numerical solution of the full Cahn-Hilliard-Navier-Stokes problem. In all works, where the phase-field method is used for the numerical simulations, either the mixture components are assumed to have matched densities (e.g. Chella \& Vinals 1996) or the solution is based on the Boussinesq approximation of the full equations (e.g. Jacqmin 1999; Ding et al. 2007; Vorobev 2010; Ahmadlouydarab \& Feng 2014; Xie \& Vorobev 2016; Vorobev \& Boghi 2016; Vorobev et al. 2017; Vorobev \& Khlebnikova 2018; Lyubimova et al. 2019).

\subsection{Phase diagram}

The phase diagram determines the states of thermodynamic equilibrium of a binary mixture. In the current work we are interested in the slowly miscible mixtures that may be either homogeneous or heterogeneous. Let us assume that the evolution of the binary mixture with the upper critical (consolute) point is studied. The components of such a mixture are fully miscible if the temperature is above the critical point (that is the binary mixture is homogeneous in equilibrium), and the components are only partially miscible if the temperature is below the critical point (the mixture is heterogeneous in equilibrium). This is a common phase behaviour for liquid/liquid binary mixtures, and the examples could be the mixtures of isobutyric acid/water or butanol/water (Pojman et al. 2006), or the water soluble polymer studied by Gan et al. (2000).

The equilibrium states of such a mixture can be defined by the free energy function that was proposed by Landau \& Lifshitz (1980) for the description of near-critical systems,

$$
f_{0}=a_{T}\left(C-C_{c}\right)^{2}+b\left(C-C_{c}\right)^{4},
$$

where $C_{c}$ is the critical concentration, and $a_{T}$ and $b$ are the phenomenological coefficients.

The classical part of the chemical potential is determined by the following function,

$$
\mu_{0}=\frac{d f_{0}}{d C}=2 a_{T}\left(C-C_{c}\right)+4 b\left(C-C_{c}\right)^{3} .
$$

If one neglects the capillary and gravity effects, then the equilibrium states of the binary 
system is determined by the equation, $\mu_{0}=0$, or

$$
\frac{a_{T}}{2 b}=-\left(C-C_{c}\right)^{2} .
$$

One notices that the $a_{T} /(2 b)$ plays the role of temperature, with the critical point given by $a_{T} /(2 b)=0$. Near the critical point $b$ is always positive, and $a_{T}$ can be either positive or negative. In equilibrium, the mixture is heterogeneous if $a_{T}$ is negative, and the mixture is homogeneous if $a_{T}$ is positive. We are interested in the process of slow mixing of two liquids, and thus the current state of the mixture is assumed to be different from the state of thermodynamic equilibrium.

We should mention that expression (2.9) was initially suggested for description of near-critical states of a mixture, i.e. when $a_{T} /(2 b)<<1$, but the use of expression (2.9) is frequently extended onto the states that are quite far from the critical point. For instance, if $a_{T} /(2 b)=-1 / 2$, expression (2.9) coincides with the double-well potential that is traditionally used for diffuse-interface modelling of mixtures of immiscible liquids (see e.g. Jacqmin 1999; Ding et al. 2007; Ahmadlouydarab \& Feng 2014; Prokopev et al. 2019) to describe very different physical effects: motion of bubbles/droplets, movements of polymerisation or solidification fronts, etc.

\subsection{Non-dimensional form of the governing equations}

Further, for convenience, we shift the reference points for the fields of concentration and density as follows, $\left(C-C_{c}\right) \rightarrow C$ and $\left(\rho-\rho_{c}\right) \rightarrow \rho$. Here $\rho_{c}$ is the density of the mixture in its critical (consolute) point.

To re-write the governing equations in the non-dimensional form we adopt the following scales for the density, chemical potential, viscosity coefficient, velocity and pulsation velocity, time, and pressure, respectively,

$$
\begin{array}{r}
{[\rho]=\rho_{c},[\mu]=\mu_{*}=b,[\eta]=\eta_{1},[u]=\left[w_{0}\right]=\mu_{*}^{1 / 2},} \\
\tau=\frac{L_{*}}{[u]}=\frac{L_{*}}{\mu_{*}^{1 / 2}},[p]=\rho_{c} \mu_{*} .
\end{array}
$$

Here $L_{*}$ is the typical size of the geometrical configuration, and $\eta_{1}$ is the viscosity of the pure component 1 .

In the non-dimensional form the governing equations read

$$
\begin{gathered}
\frac{\partial \rho}{\partial t}+(\boldsymbol{v} \cdot \nabla) \rho=-(1+\rho) \nabla \cdot \boldsymbol{v} \\
(1+\rho)\left(\frac{\partial C}{\partial t}+(\boldsymbol{v} \cdot \nabla) C\right)=\frac{1}{P e} \nabla^{2} \mu \\
(1+\rho)\left(\frac{\partial \boldsymbol{v}}{\partial t}+(\boldsymbol{v} \cdot \nabla) \boldsymbol{v}\right)=-\nabla p+\frac{1}{R e} \nabla \cdot \tau_{\eta}-C n \nabla \cdot \tau_{\epsilon}-G a(1+\rho) \boldsymbol{\gamma}- \\
-(1+\rho) \frac{\partial \boldsymbol{w}_{0}}{\partial t} .
\end{gathered}
$$

The equation of state is given by

$$
\rho=\frac{\phi C}{1-\phi C} ; \phi=\frac{\rho_{c}\left(\rho_{02}-\rho_{01}\right)}{\rho_{01} \rho_{02}} .
$$

Here $\phi$ is the density contrast for the mixture. For all liquid/liquid mixtures $\phi$ is rather small, and thus equation (2.17) could be further simplified e.g. by keeping only the linear term in the expansion over small $\phi$. 
The chemical potential is defined by the equation

$$
\mu=2 A C+4 C^{3}-\phi p-C n\left(\nabla^{2} C+\phi(1+\rho)(\nabla C)^{2}\right) .
$$

The viscous stress tensor and the Korteweg stress tensor are determined by the following expressions

$$
\begin{gathered}
\tau_{\eta}=\eta\left(\nabla \otimes \boldsymbol{v}+(\nabla \otimes \boldsymbol{v})^{T}-\frac{2}{3}(\nabla \cdot \boldsymbol{v}) I\right), \\
\tau_{\epsilon}=(1+\rho) \nabla C \otimes \nabla C .
\end{gathered}
$$

The above equations include a number of the non-dimensional parameters. These are the Reynolds and Péclet numbers,

$$
R e=\frac{\rho_{*} \mu_{*}^{1 / 2} L_{*}}{\eta_{*}}, P e=\frac{\rho_{*} L_{*}}{\alpha \mu_{*}^{1 / 2}},
$$

the Galileo number and the Cahn number (or the capillary parameter)

$$
G a=\frac{g L_{*}}{\mu_{*}}, C n=\frac{\epsilon}{\mu_{*} L_{*}^{2}},
$$

the parameter that determines the thermodynamic states of the mixture (this parameter can be interpreted as the temperature of the binary mixture)

$$
A=\frac{a_{T}}{b},
$$

and the non-dimensional frequency of the vibrational forcing,

$$
\Omega=\frac{\omega L_{*}}{\mu_{*}^{1 / 2}} .
$$

All these parameters are introduced on the basis of the non-traditional phenomenological coefficients that are adopted by the phase-field approach. We keep using the standard names for the non-dimensional parameters as almost all of them appear in front of the corresponding terms of the governing equations, and thus their values determine the corresponding physical effects (e.g. the Péclet number determines the role of the diffusive mass transport, the Reynolds number defines the role of the viscous force, the Galileo number sets the level of the gravity force). The Cahn (capillary) number determines the role of the surface tension effects.

\section{Multiple-scale analysis}

\subsection{Typical time scales}

The physical processes defined by equations (2.14)-(2.20) are characterized by the different time scales. These are the diffusion and convection scales

$$
\tau_{d}=\frac{P e}{|A|}, \tau_{c}=R e,
$$

the vibration scale (the period of vibrations),

$$
\tau_{v}=\frac{1}{\Omega},
$$


and the expansion time scale that characterises the duration of the quasi-acoustic processes,

$$
\tau_{e}=\frac{\phi^{2}}{P e}
$$

Two vertical lines in (3.1) denote the absolute value.

We develop the theory for the high-frequency vibrations, when $\tau_{v}<<\left(\tau_{d}, \tau_{c}\right)$, i.e. the period of vibrations is much shorter than the time periods needed for the diffusive and convective changes. The validity of this assumption is easy to illustrate, as $\tau_{v} / \tau_{c}=$ $\eta_{*} /\left(\rho_{*} \omega L_{*}^{2}\right)$ and $\tau_{v} / \tau_{d}=\left(\alpha \mu_{*}\left|a_{T}\right|\right) /\left(\rho_{*} \omega L_{*}^{2}\right) \sim\left(D_{*}\left|a_{T}\right|\right) /\left(\omega L_{*}^{2}\right)$, and thus both ratios are indeed very small if $\omega>>\eta_{*} /\left(\rho_{*} L_{*}^{2}\right)$ and $\omega>>D_{*} / L_{*}^{2}$, or, in other words, $\omega$ is greater than, at least, $0.01 \mathrm{~s}^{-1}$; if one assumes that fluid's density $\rho \sim 10^{3} \mathrm{~kg} \cdot \mathrm{m}^{-3}$, viscosity coefficient $\eta_{*} \sim 10^{-3} \mathrm{~Pa} \cdot \mathrm{s}$, diffusion coefficient $D_{*} \sim 10^{-9} \mathrm{~m}^{2} \mathrm{~s}^{-1}$, and the typical size of geometry $L_{*} \sim 10^{-2} \mathrm{~m}$.

In addition, typically, $R e>>1$ and $P e>>1$, and $\phi<<1$, which means that the expansion time scale is also small, $\tau_{e}<<\left(\tau_{d}, \tau_{c}\right)$. Some ambiguity although remains regarding the relation between the vibration and expansion time scales. We want to separate these processes, and assume that the expansion scale is much smaller than the period of the vibrational forcing. Thus, the following inequalities are implied for our further analysis,

$$
\tau_{e}<<\tau_{v}<<\left(\tau_{d}, \tau_{c}\right) .
$$

Following the main idea of the multiple scale method we introduce the three different times: the fastest time, $t_{-2}$, to describe the quick quasi-acoustic processes, the intermediate time, $t_{0}$, for description of the pulsation processes, and the slow time, $t_{2}$, for the description of the diffusion and convection processes. The time derivative is then replaced by a sum of three terms,

$$
\frac{\partial}{\partial t}=\chi^{-2} \frac{\partial}{\partial t_{-2}}+\frac{\partial}{\partial t_{0}}+\chi^{2} \frac{\partial}{\partial t_{2}}
$$

Here $\chi$ is a small parameter.

In addition, all physical variables are also expanded into the series of the same small parameter $\chi$,

$$
\begin{array}{r}
\boldsymbol{v}=\chi^{2} \boldsymbol{v}_{2}+\chi^{4} \boldsymbol{v}_{4}+\ldots, \\
p=p_{0}+\chi^{2} p_{2}+\chi^{4} p_{4}+\ldots, \\
\mu=\chi \mu_{1}+\chi^{3} \mu_{3}+\ldots, \\
\rho=\chi^{2} \rho_{2}+\chi^{4} \rho_{4}+\ldots, \\
C=\chi C_{1}+\chi^{3} C_{3}+\ldots, \\
\eta=1+\ldots
\end{array}
$$

The viscosity coefficient is assumed to be a regular function of concentration. The expansion of this function starts from 1 due to adopted scaling (2.12). For our analysis however the higher orders of this expansion are not needed.

In addition, we make the following assumptions about the values of the non-dimensional parameters,

$$
\phi=\chi \phi_{1}, P e=P e_{0}, R e=\chi^{-2} R e_{-2}, G a=\chi^{2} G a_{2}, C n=\chi^{2} C n_{2}, A=\chi^{2} A_{2} .
$$

These assumptions are primarily written on the basis of the two requirements: (i) the final theoretical model should incorporate all relevant physical effects, and the final theoretical 
model should be self-consistent, with no terms that demand the knowledge of the higherorder variables thus requiring further phenomenological modelling.

\subsection{Different orders of the governing equations}

Next, using the assumptions summarized above, we write the different orders of the governing equations that define the evolution of the mixture. The first two orders of the continuity equation read:

$$
\begin{array}{r}
\frac{\partial \rho_{2}}{\partial t_{-2}}=0 \\
\frac{\partial \rho_{4}}{\partial t_{-2}}+\frac{\partial \rho_{2}}{\partial t_{0}}=-\nabla \cdot \boldsymbol{v}_{2}
\end{array}
$$

The first equation, in particular, states that $\rho_{2}$ (the highest order of the density field) does not depend on the fastest time scale.

For the equation for the species conservation the first three orders are written,

$$
\begin{array}{r}
\frac{\partial C_{1}}{\partial t_{-2}}=0, \\
\frac{\partial C_{3}}{\partial t_{-2}}+\frac{\partial C_{1}}{\partial t_{0}}=\frac{1}{P e_{0}} \nabla^{2} \mu_{1}, \\
\frac{\partial C_{5}}{\partial t_{-2}}+\frac{\partial C_{3}}{\partial t_{0}}+\frac{\partial C_{1}}{\partial t_{2}}+\left(\boldsymbol{v}_{2} \cdot \nabla\right) C_{1}+\rho_{2}\left[\frac{\partial C_{3}}{\partial t_{-2}}+\frac{\partial C_{1}}{\partial t_{0}}\right]=\frac{1}{P e_{0}} \nabla^{2} \mu_{3} .
\end{array}
$$

Finally, the first three orders of the momentum-balance equation read

$$
\begin{array}{r}
\frac{\partial \boldsymbol{v}_{2}}{\partial t_{-2}}=-\nabla p_{0}-\frac{\partial \boldsymbol{w}_{0}}{\partial t_{0}} \\
\frac{\partial \boldsymbol{v}_{4}}{\partial t_{-2}}+\frac{\partial \boldsymbol{v}_{2}}{\partial t_{0}}+\rho_{2} \frac{\partial \boldsymbol{v}_{2}}{\partial t_{-2}}=-\nabla p_{2}-G a_{2} \gamma-\rho_{2} \frac{\partial \boldsymbol{w}_{0}}{\partial t_{0}} \\
\frac{\partial \boldsymbol{v}_{6}}{\partial t_{-2}}+\frac{\partial \boldsymbol{v}_{4}}{\partial t_{0}}+\frac{\partial \boldsymbol{v}_{2}}{\partial t_{2}}+\left(\boldsymbol{v}_{2} \cdot \nabla\right) \boldsymbol{v}_{2}+\rho_{2}\left[\frac{\partial \boldsymbol{v}_{4}}{\partial t_{-2}}+\frac{\partial \boldsymbol{v}_{2}}{\partial t_{0}}+\rho_{2} \frac{\partial \boldsymbol{v}_{2}}{\partial t_{-2}}\right]=-\nabla p_{4}+ \\
+\frac{1}{R e_{-2}} \nabla \cdot \tau_{\eta, 2}-C a_{2} \nabla \cdot \tau_{\epsilon, 2}-G a_{2} \rho_{2} \gamma-\rho_{4} \frac{\partial \boldsymbol{w}_{0}}{\partial t_{0}}
\end{array}
$$

Here,

$$
\begin{array}{r}
\tau_{\eta, 2}=\nabla \otimes \boldsymbol{v}_{2}+\left(\nabla \otimes \boldsymbol{v}_{2}\right)^{T}-\frac{2}{3}\left(\nabla \cdot \boldsymbol{v}_{2}\right) I \\
\tau_{\epsilon, 2}=\nabla C_{1} \otimes \nabla C_{1} .
\end{array}
$$

\subsection{Averaging}

As mentioned above, it is assumed that the evolution of the mixture can be represented by some rapid changes that occur on the background of the slower processes. Thus, all variables can be further split into the following sums,

$$
h\left(t_{-2}, t_{0}, t_{2}\right)=\bar{h}\left(t_{2}\right)+\widetilde{h}\left(t_{0}, t_{2}\right)+\widetilde{\widetilde{h}}\left(t_{-2}, t_{0}, t_{2}\right) .
$$

Here $h$ stands for any variable. The averaging procedure is used to obtain the evolution of the mixture on a longer time scale. For instance, to get rid of the fast quasi-acoustic processes, the equations can be averaged over the period of vibrations, $T_{0} \equiv 1 / \Omega$,

$$
\widetilde{h}\left(t_{0}, t_{2}\right)=\frac{1}{T_{0}} \int_{T_{0}} \widetilde{\widetilde{h}}\left(t_{-2}, t_{0}, t_{2}\right) \mathrm{d} t_{-2} .
$$


Similarly, the dynamics on the diffusive and convective time scales can be singled out by averaging of the equations over the time period that is much greater than the period of the vibrations. To obtain the equations for the faster processes the averaged equations need to be subtracted from the full governing equations.

We distinguish the variables that determine the average, pulsation and quasi-acoustic processes by adding the double-tilde for the quasi-acoustic parts, the single tilde for the vibrational oscillations, and the bar to denote the averaged quantities. The pulsational fluid velocity is denoted by the vector $\boldsymbol{w}$ and the velocity field that characterises the convective processes is denoted by the vector $\boldsymbol{u}$.

With the help of the outlined averaging procedure, the following equations are obtained to determine the fastest quasi-acoustic processes,

$$
\begin{array}{r}
\frac{\partial \widetilde{\widetilde{\boldsymbol{v}}}_{2}}{\partial t_{-2}}=-\nabla \widetilde{\widetilde{p}}_{0}, \\
\frac{\partial \widetilde{\widetilde{C}}_{3}}{\partial t_{-2}}=\frac{1}{P e_{0}} \nabla^{2} \widetilde{\widetilde{\mu}}_{1}, \frac{\partial \widetilde{\widetilde{\rho}}_{4}}{\partial t_{-2}}=-\nabla \cdot \widetilde{\widetilde{\boldsymbol{v}}}_{2}, \\
\widetilde{\widetilde{p}}_{0}=-\frac{1}{\phi_{1}} \widetilde{\widetilde{\mu}}_{1}, \widetilde{\widetilde{\rho}}_{4}=\phi_{1} \widetilde{\widetilde{C}}_{3} .
\end{array}
$$

These equations define the rapidly decaying processes, so that all temporal and spatial variations decay exponentially, as $\sim \exp \left(-\frac{P e_{0}}{\phi_{1}^{2}} t_{-2}\right)$. Thus, even if at some time moment, such processes are excited in a system, they rapidly die out leaving no influence on the slower dynamics.

The equations that determine the pulsation fields are

$$
\begin{array}{r}
\frac{\partial \boldsymbol{w}_{2}}{\partial t_{0}}=-\nabla \widetilde{p}_{2}-\widetilde{\rho}_{2} \frac{\partial \boldsymbol{w}_{0}}{\partial t_{0}}, \\
\frac{\partial \widetilde{\rho}_{2}}{\partial t_{0}}=-\nabla \cdot \boldsymbol{w}_{2}, \\
\frac{\partial \widetilde{C}_{1}}{\partial t_{0}}=\frac{1}{P e_{0}} \nabla^{2} \widetilde{\mu}_{1} .
\end{array}
$$

Using the corresponding order of the equation of state, $\rho_{2}=\phi_{1} C_{1}$, and the corresponding order of the expression for the chemical potential, $\mu_{1}=-\phi_{1} p_{0}$, these equations can be re-written as

$$
\begin{aligned}
\nabla \times \boldsymbol{w}_{2} & =\boldsymbol{w}_{0} \times \nabla \rho_{2}, \\
\nabla \cdot \boldsymbol{w}_{2} & =\frac{\phi_{1}^{2}}{P e_{0}} \nabla^{2} \widetilde{p}_{0} .
\end{aligned}
$$

Averaging of (3.18) over the period of vibrations gives the following relation for the pulsation part of the pressure field,

$$
\nabla \widetilde{p}_{0}+\frac{\partial \boldsymbol{w}_{0}}{\partial t_{0}}=0
$$

Applying the divergence to the above equation shows that $\nabla^{2} \widetilde{p}_{0}=0$, and hence $\nabla^{2} \widetilde{\mu}_{1}=$ 0 . Thus, finally, we conclude that the pulsation fields are determined by the following equations:

$$
\frac{\partial \widetilde{\rho}_{2}}{\partial t_{0}}=0, \frac{\partial \widetilde{C}_{1}}{\partial t_{0}}=0
$$




$$
\begin{array}{r}
\nabla \times \boldsymbol{w}_{2}=\boldsymbol{w}_{0} \times \nabla \rho_{2}, \\
\nabla \cdot \boldsymbol{w}_{2}=0 .
\end{array}
$$

Earlier we noted that $\rho_{2}$ does not depend on $t_{-2}$, and the first equation (3.34) states that $\rho_{2}$ does not depend on the pulsation time $t_{0}$ either. Thus the leading orders of the density and concentration fields $\left(\rho_{2}\right.$ and $\left.C_{1}\right)$ depend only on the slow time $t_{2}$, and further on we cease using the bar for the averaged parts of these variables (as the other parts for these variables are non-existent).

Let us now write the equations for the slow evolution on the diffusive and convective time scales,

$$
\begin{gathered}
\frac{\partial \boldsymbol{u}_{2}}{\partial t_{2}}+\left(\boldsymbol{u}_{2} \cdot \nabla\right) \boldsymbol{u}_{2}+\overline{\left(\boldsymbol{w}_{2} \cdot \nabla\right) \boldsymbol{w}_{2}}=-\nabla \bar{p}_{4}+\frac{1}{R e}{ }_{-2} \nabla \cdot \bar{\tau}_{\eta, 2}-C n_{2} \nabla \cdot \bar{\tau}_{\epsilon, 2}- \\
-G a_{2} \rho_{2} \gamma-\overline{\tilde{\rho}_{4} \frac{\partial \boldsymbol{w}_{0}}{\partial t_{0}}} \\
\frac{\partial C_{1}}{\partial t_{2}}+\left(\boldsymbol{u}_{2} \cdot \nabla\right) C_{1}=\frac{1}{P e_{0}} \nabla^{2} \bar{\mu}_{3}, \\
\nabla \cdot \boldsymbol{u}_{2}=0 .
\end{gathered}
$$

Here,

$$
\bar{\tau}_{\eta, 2}=\nabla \otimes \boldsymbol{u}_{2}+\left(\nabla \otimes \boldsymbol{u}_{2}\right)^{T}, \bar{\tau}_{\epsilon, 2}=\nabla C_{1} \otimes \nabla C_{1} .
$$

There are two additional terms in the momentum balance equation (3.37) that appear due to the averaging of the non-linear terms. The first term can be re-written as follows,

$$
\begin{aligned}
\left(\boldsymbol{w}_{2} \cdot \nabla\right) \boldsymbol{w}_{2}=\nabla \frac{\boldsymbol{w}_{2}^{2}}{2}-\boldsymbol{w}_{2} & \times\left(\nabla \times \boldsymbol{w}_{2}\right)=\nabla \frac{\boldsymbol{w}_{2}^{2}}{2}-\boldsymbol{w}_{2} \times\left(\boldsymbol{w}_{0} \times \nabla \rho_{2}\right)= \\
& =\nabla \frac{\boldsymbol{w}_{2}^{2}}{2}-\left(\boldsymbol{w}_{0}\left(\boldsymbol{w}_{2} \cdot \nabla \rho_{2}\right)-\left(\boldsymbol{w}_{2} \cdot \boldsymbol{w}_{0}\right) \nabla \rho_{2}\right) .
\end{aligned}
$$

Here equation (3.35) is used. The term in the right hand side can be also modified as,

$$
\overline{\widetilde{\rho}_{4} \frac{\partial \boldsymbol{w}_{0}}{\partial t_{0}}}=-\overline{\boldsymbol{w}_{0} \frac{\partial \widetilde{\rho}_{4}}{\partial t_{0}}} .
$$

To modify $\frac{\partial \widetilde{\rho}_{4}}{\partial t_{0}}$ we use equation (3.16) together with the corresponding orders of the equation of state, $\widetilde{\rho}_{4}=\phi_{1} \widetilde{C}_{3}$ and of the equation for the chemical potential, $\widetilde{\mu}_{3}=-\phi_{1} \widetilde{p}_{2}$, thus obtaining

$$
\frac{\partial \widetilde{\rho}_{4}}{\partial t_{0}}=-\left(\boldsymbol{w}_{2} \cdot \nabla\right) \rho_{2}+\frac{\phi_{1}^{2}}{P e_{0}}\left(\nabla \rho_{2} \cdot \frac{\partial \boldsymbol{w}_{0}}{\partial t_{0}}\right) .
$$

Substitution of this equation into (3.42) gives

$$
\overline{\tilde{\rho}_{4} \frac{\partial \boldsymbol{w}_{0}}{\partial t_{0}}}=\overline{\boldsymbol{w}_{0}\left(\boldsymbol{w}_{2} \cdot \nabla \rho_{2}\right)} .
$$

Hence, the equations for the diffusive and convective evolution of the system have the following view

$$
\begin{aligned}
\frac{\partial \boldsymbol{u}_{2}}{\partial t_{2}}+\left(\boldsymbol{u}_{2} \cdot \nabla\right) \boldsymbol{u}_{2}= & -\nabla\left(\bar{p}_{4}+\frac{\overline{\boldsymbol{w}_{2}^{2}}}{2}+C n_{2} \frac{\left(\nabla C_{1}\right)^{2}}{2}\right)+\frac{1}{R e_{-2}} \nabla^{2} \boldsymbol{u}_{2}- \\
& -C n_{2} \nabla^{2} C_{1} \nabla C_{1}-\phi_{1} G a_{2} C_{1} \boldsymbol{\gamma}-\phi_{1} \overline{\left(\boldsymbol{w}_{2} \cdot \boldsymbol{w}_{0}\right)} \nabla C_{1},
\end{aligned}
$$




$$
\begin{gathered}
\frac{\partial C_{1}}{\partial t_{2}}+\left(\boldsymbol{u}_{2} \cdot \nabla\right) C_{1}=\frac{1}{P e_{0}} \nabla^{2} \bar{\mu}_{3}, \\
\nabla \cdot \boldsymbol{u}_{2}=0 .
\end{gathered}
$$

The equation for the averaged part of the chemical potential reads

$$
\bar{\mu}_{3}=\phi_{1} G a_{2}(\boldsymbol{\gamma} \cdot \boldsymbol{r})+2 A_{2} C_{1}+4 C_{1}^{3}-C n_{2} \nabla^{2} C_{1} .
$$

And the equations for the pulsation fields are

$$
\begin{array}{r}
\nabla \times \boldsymbol{w}_{2}=\phi_{1} \boldsymbol{w}_{0} \times \nabla C_{1}, \\
\nabla \cdot \boldsymbol{w}_{2}=0 .
\end{array}
$$

Equations (3.45)-(3.50) define a closed self-consistent set of the equations that provide the effective description of the evolution of the binary mixture that is subjected to the high-frequency vibrational forcing. In particular, equation (3.45) includes two terms of the vibrational origin that appear due to the averaging of the non-linear terms of the Navier-Stokes equation. These are the gradient term, $\nabla\left(\overline{\boldsymbol{w}_{2}^{2}} / 2\right)$, that does not affect fluid motion in a closed container, but may be essential for description of a system with a free interface; and the vibrational force, $\phi_{1} \overline{\left(\boldsymbol{w}_{2} \cdot \boldsymbol{w}_{0}\right)} \nabla C_{1}$, that determines the volumetric mechanism for the generation of the averaged flow.

\subsection{Boundary conditions}

The high-frequency vibrations are known to generate thin skin-layers near the rigid walls and near interfaces (see Lyubimov et al. 2003). Thus, the problem is additionally characterised by the small length scales that can be estimated as $\delta_{c} \sim 1 / \sqrt{R e \Omega}$ or $\delta_{d} \sim$ $\sqrt{|A| /(P e \Omega)}$, which are the thicknesses of the viscous and diffusive layers, respectively.

Let us analyse the behaviour of all variables within the skin-layer that is formed near the wall (the wall's position is to be given by $z=0$ with $z$ being the coordinate perpendicular to the wall). For the analysis, we introduce two spatial coordinates, $z_{-1}$ to track the faster changes within the skin-layer and $z_{0}$ to describe the slow changes on the longer scale. In accordance with the multiple-scale method, the $z$-derivative is represented as

$$
\frac{\partial}{\partial z}=\chi^{-1} \frac{\partial}{\partial z_{-1}}+\frac{\partial}{\partial z_{0}}
$$

For convenience, within the vibration skin-layer the velocity is denoted by $\boldsymbol{v}=\left(\boldsymbol{V}, v_{z}\right)$, where $\boldsymbol{V}$ and $v_{z}$ stand for the velocity components tangential and perpendicular to the wall (correspondingly, $\left(\boldsymbol{W}, w_{z}\right)$ and $\left(\boldsymbol{U}, u_{z}\right)$ are used for the pulsation and averaged velocities), and the nabla-operator is denoted as $\nabla=\left(\nabla_{2}, \partial / \partial z\right)$, where $\nabla_{2}$ includes the derivatives along the wall, and $\partial / \partial z$ is the derivative normal to the wall.

Next, by using expansions (3.6) and assumptions (3.12), we write the leading orders of the governing equations for the skin-layer. The first two orders of the continuity equation are

$$
\frac{\partial \rho_{2}}{\partial t_{-2}}=0, \frac{\partial v_{z, 2}}{\partial z_{-1}}=0
$$

Thus the normal derivative of the velocity vector is independent of the quick coordinate. Since the normal component of the velocity is zero at the wall, it remains zero within the skin-layer. This statement is true for both the pulsation and averaged velocities.

The leading orders of the equation for the $z$-component of the momentum-balance equation state that

$$
\frac{\partial p_{0}}{\partial z_{-1}}=0, \frac{\partial p_{2}}{\partial z_{-1}}=0
$$


which signify that the highest orders of the pressure do not depend on the fast coordinate.

The leading orders of the equation for the momentum balance along the wall is

$$
\begin{array}{r}
\frac{\partial \boldsymbol{V}_{2}}{\partial t_{-2}}=-\nabla_{2} p_{0}-\frac{\partial \boldsymbol{w}_{0}}{\partial t_{0}}, \\
\frac{\partial \boldsymbol{V}_{4}}{\partial t_{-2}}+\frac{\partial \boldsymbol{V}_{2}}{\partial t_{0}}=-\nabla_{2} p_{2}+\frac{1}{R e_{-2}} \frac{\partial^{2} \boldsymbol{V}_{2}}{\partial z_{-1}^{2}}-\rho_{2} \frac{\partial \boldsymbol{w}_{0}}{\partial t} .
\end{array}
$$

The averaging of the latest equation gives

$$
-\nabla_{2} \bar{p}_{2}+\frac{1}{R e_{-2}} \frac{\partial^{2} \boldsymbol{U}_{2}}{\partial z_{-1}^{2}}=0
$$

Since the term $\nabla_{2} \bar{p}_{2}$ is independent of $z_{-1}$, it needs to be equal zero, otherwise the tangential component of the averaged velocity diverge as $\sim z_{-1}$. Then, owing to the noslip boundary condition that is imposed at the wall, one concludes that the tangential component of the averaged velocity is zero within the entire pulsation skin-layer.

The leading orders of the equation for the species conservation read

$$
\frac{\partial^{2} \mu_{1}}{\partial z_{-1}^{2}}=0, \frac{\partial C_{3}}{\partial t_{-2}}+\frac{\partial C_{1}}{\partial t_{0}}=\frac{1}{P e_{0}}\left(\nabla_{2}^{2} \mu_{1}+\frac{\partial^{2} \mu_{3}}{\partial z_{-1}^{2}}\right) .
$$

Here we already used that the leading order of density (and hence concentration) does not depend on the fast time-scale, $t_{-2}$.

The first order of the equation for the chemical potential reads

$$
\mu_{1}=-\phi_{1} p_{0}-C n_{2} \frac{\partial^{2} C_{1}}{\partial z_{-1}^{2}} .
$$

The no-penetration condition, stated as the absence of the normal component of the chemical potential at the wall, together with equations (3.53), (3.57), and (3.58), allow us to conclude that within the skin-layer

$$
\frac{\partial \mu_{1}}{\partial z_{-1}}=0, \frac{\partial C_{1}}{\partial z_{-1}}=0
$$

The second condition is required as otherwise the values of $C_{1}$ would diverge. Further, averaging of the second equation (3.57) gives us the statement that the normal derivative of the chemical potential is zero within the skin-layer

$$
\frac{\partial \bar{\mu}_{3}}{\partial z_{-1}}=0
$$

Summarising, we conclude at the outer side of the skin-layer,

$$
\boldsymbol{U}_{2}=0, U_{z, 2}=w_{z, 2}=0 .
$$

Thus, in our case, there is no generation of the averaged flow at the wall, which however is the general case for the spatially uniform vibrations (see Gershuni \& Lyubimov 1998).

In addition, the highest orders of the concentration and density fields, $C_{1}$ and $\rho_{2}$, and the averaged part of the chemical potential, $\bar{\mu}_{3}$ are independent of the quick coordinate, which mean that the requirements imposed on the values of the chemical potential and concentration on the wall (i.e. the zero normal derivative of the chemical potential and e.g. zero normal derivative of the concentration) remain unchanged within the vibration skinlayer. There are no averaged effects induced at the walls. Nevertheless, the undertaken analysis is still useful as the derived conditions make unnecessary the resolution of the vibration skin-layers. 


\section{Discussion and conclusions}

We now omit the indexes that were used to denote the orders of the variables and re-write the resultant equations (3.45)-(3.50) in the dimensional form.

The equations for the slow dynamics of the binary mixture read

$$
\begin{gathered}
\frac{\partial \boldsymbol{u}}{\partial t}+(\boldsymbol{u} \cdot \nabla) \boldsymbol{u}=-\nabla\left(\frac{\Pi}{\rho}\right)+\frac{\eta}{\rho} \nabla^{2} \boldsymbol{u}-\epsilon \nabla^{2} C \nabla C+\phi C \boldsymbol{g}-\frac{\phi}{2}\left(\boldsymbol{w} \cdot \boldsymbol{w}_{\mathbf{0}}\right) \nabla C, \\
\frac{\partial C}{\partial t}+(\boldsymbol{u} \cdot \nabla) C=\frac{\alpha}{\rho} \nabla^{2} \mu, \\
\nabla \cdot \boldsymbol{u}=0 .
\end{gathered}
$$

In the equation for the species conservation (4.2), the letter $\mu$ denotes the average part of the chemical potential, that is determined by the following expression

$$
\mu=-\phi(\boldsymbol{g} \cdot \boldsymbol{r})+2 a_{T} C+4 b C^{3}-\epsilon \nabla^{2} C .
$$

The letter $\Pi$ is used to denote the modified averaged pressure,

$$
\Pi=p+\rho\left(\frac{\boldsymbol{w}^{2}}{4}+\epsilon \frac{(\nabla C)^{2}}{2}\right) .
$$

The equation for the momentum balance (4.1) includes the vibrational force that can obtained by solving the following equations for the pulsation flow:

$$
\begin{array}{r}
\nabla \times \boldsymbol{w}=\phi \boldsymbol{w}_{0} \times \nabla C, \\
\nabla \cdot \boldsymbol{w}=0 .
\end{array}
$$

Here $\boldsymbol{w}_{0}=a \omega \boldsymbol{j}$ is the amplitude of the imposed vibrational forcing.

It is useful also to note that the Navier-Stokes equation (4.1) can be re-written as

$$
\frac{\partial \boldsymbol{u}}{\partial t}+(\boldsymbol{u} \cdot \nabla) \boldsymbol{u}=-\nabla\left(\frac{\Pi_{1}}{\rho}\right)+\frac{\eta}{\rho} \nabla^{2} \boldsymbol{u}-C \nabla \mu-\frac{\phi}{2}\left(\boldsymbol{w} \cdot \boldsymbol{w}_{0}\right) \nabla C,
$$

where

$$
\Pi_{1}=p+\rho\left(\frac{\boldsymbol{w}^{2}}{4}+\epsilon \frac{(\nabla C)^{2}}{2}-\mu C+f_{0}-\phi(\boldsymbol{g} \cdot \boldsymbol{r}) C\right) .
$$

The latest form for the Korteweg force, $-C \nabla \mu$, is used in the majority of the works that utilise the phase-field approach for the numerical studies (e.g. Jacqmin 1999; Ding et al. 2007; Ahmadlouydarab \& Feng 2014). It is necessary to note that the buoyancy term in equation (4.8) is now hidden in the Korteweg term, and this term can be explicitly written if expression (4.4) is substituted into equation (4.8).

The derived set of the governing equations needs to be supplemented with the boundary conditions. For the average velocity, the no-slip boundary condition needs to be imposed, $\boldsymbol{u}=0$, stating that both the tangential and normal components of the average velocity are zero at the rigid walls. The differential equations that define the pulsation field are of the lower order, thus requiring only the condition, that the normal component of the pulsation velocity is zero, to be imposed at the wall (this condition reflects the no-penetration of the mixture through the wall), $w_{n}=0$.

Additionally, the zero value of the normal derivative of the chemical potential, $(\partial \mu) /(\partial n)=0$, needs to be imposed to exclude the diffusive flux through the wall. Finally, since the obtained equations are of the forth order in terms of concentration, the additional condition is needed. This additional condition defines the wetting properties of the wall. There are two simplest cases of the wetting properties, (i) the walls are neutral 
to the molecules of the mixture components, and thus the contact line is orthogonal to the wall, $(\partial C) /(\partial n)=0$, and (ii) the wall is perfectly wetted by one of the liquids, and in this case the value of the concentration that corresponds to the component needs to be maintained at the wall.

Let us summarise the assumptions that we make to derive equations (4.1)-(4.6). We assume that the vibrations are set by a periodic function. The amplitude of the vibrations is spatially uniform (if the fluid is enclosed in an oscillating container then all walls of the container oscillate with the same amplitude). The period of the vibrations is much smaller than the typical convective and diffusive time scales, $\omega>>\eta_{*} /\left(\rho_{*} L_{*}^{2}\right)$ and $\omega>>D_{*} / L_{*}^{2}$. At the same time the expansion time scale remains much smaller than the period of vibrations, or $\omega<<\rho_{*} /\left(\alpha \phi^{2}\right)$. The density contrast is assumed to be small, $\phi<<1$; the mobility coefficient is constant; the dependence of the viscosity on concentration is given by a regular function that may be expanded into a Taylor series near the critical (consolute) point. The classical part of the free energy function is approximated by the Landau formula (2.9), i.e. by two terms with comparable values. The binary mixture remains isothermal, which includes the assumption that the latent heat of mixing is negligibly small.

The final equations (4.1)-(4.4) are similar to the Boussinesq approximation of the full Cahn-Hilliard-Navier-Stokes equations, that was earlier derived using the similar multiple-scale analysis developed by Vorobev (2010). The obtained theoretical model describes the slow diffusive and convective evolution of the binary mixture with the account of the dynamic (varying over the duration of the process) surface tension effects. The diffusion term is defined on the basis of the extended Fick's law, and thus the model includes the effect of barodiffusion. If, however, expression (4.4) is substituted into the species balance equation, then the barodiffusive term drops out. Nevertheless, this term still affects the boundary conditions, and thus influencing the equilibrium states of the mixture and the long-term dynamics of the mixture when the influence of the boundary conditions propagates into the bulk. In particular, this term, introduces the density stratification in a homogeneous mixture, and this term defines the deformation of the shapes of interfacial boundaries by e.g. the action of the gravity force.

The difference of the new model from the earlier equations (Vorobev 2010) is the addition of the vibration terms. The averaged vibrational force and the equations for the pulsation flow coincide with the standard equations that determine the effects of the uniform vibrations on a single-phase fluid flow (see e.g. Gershuni \& Lyubimov 1998; Gaponenko et al. 2006; Gaponenko \& Shevtsova 2010). It is known that the vibrational terms are different for the cases of the uniform and non-uniform vibrational fields. The imposed vibrational forcing is spatially uniform, although, it is also known that the vibration field becomes spatially non-uniform in a heterogeneous fluid with the interfaces that separate different phases. In the case of the non-uniform vibrations the vibrational terms in the averaged equations are different (some of the terms, that dropped out during the derivation of the averaged equations shown in this work, would stay, and these terms appear in the different orders of the multiple-scale analysis (see e.g. Lyubimov et al. $2006 a, b)$ ). We model the behaviour of the heterogeneous system, and thus should expect the similarity of the resultant equations with the non-uniform model. Although, seemingly owing to the chosen phase-field approach, and owing to the assumptions made, which effectively imply the consideration of a near-critical system when the thickness of an interfacial boundary is not small, the final equations coincide with the standard averaged model for the uniform vibrations.

The advantage introduced by the obtained model is the possibility to perform the time-integration with the larger time steps, with no need for the resolution of the quick 
quasi-acoustic and pulsation effects that would require the use of smaller time steps. The use of the effective boundary conditions also permits not to resolve the thin vibrational skin-layers. Although, owing to the diffuse interface approach that is adopted for the description of the multi-phase system, the increased spatial resolution required for the resolution of the interfacial boundaries is still needed.

The real thickness of an interface is just several molecular layers $(\sim 1 \mathrm{~nm})$, which is zero for a macroscopic approach. The interface is smeared (replaced by a transitional layer of a finite thickness) primarily for the sake of numerical description. In order to obtain the physically-relevant results one needs to establish the behaviour of a multiphase system in the limit of infinitely thin interface, which is possible to obtain by running the simulations for different values of the interface thickness and observing the limiting behaviour of a multiphase system. Frequently, this is a computational consuming task, although, this requirement applies to any diffuse-interface numerical approach.

We should also mention that the main advantage of the model is that the set of obtained equations is closed, and now this model could be used for a variety of different problems. Moreover, the applicability of the derived equations should not be strictly restricted to the assumptions made during the derivation (i.e. to the vicinity to the critical point). The standard Boussinesq approximation, for instance, is also derived in the assumptions of smaller values of the density contrast and larger values of the Galileo number, so that their product, the Rayleigh number, remains finite. Later, however, the Boussinesq equations are frequently used for quite large values of the Rayleigh numbers. The Landau free energy function, that we use to determine the thermodynamic model of the binary mixture, was originally proposed for near critical systems, but this function is in fact coincide with the 'double-well' potential (see Jacqmin 1999; Ding et al. 2007; Ahmadlouydarab \& Feng 2014) that is traditionally utilised for tracing the deformations of immiscible interfaces (i.e. far from the critical point). Finally, we should mention that the obtained model includes just one viscosity coefficient, which however does not imply that the viscosity of the mixture is constant. As mentioned in the main part of the work, we assume that the viscosity is a regular function of concentration, although for the assumptions made, the leading order of the viscous force does not require the account of the variations in the viscosity coefficient.

In the end, let us illustrate the use of the obtained equations for a very simple problem. Namely, we obtain the state of quasi-equilibrium for two semi-infinite liquid domains that are brought into contact. In the theory of vibrational convection (Gershuni \& Lyubimov 1998) the state of quasi-equilibrium is determined as the state with no averaged flows (i.e. $\boldsymbol{u}=0$ ), but with the non-zero pulsation motion. For simplicity, we neglect the gravity effect, and consider the $2 D$ solution only, introducing the Cartesian coordinates $x$ and $y$ to characterise the spatial changes in the system along and across the liquid/liquid interface. The equations that determine the quasi-equilibrium state read

$$
\begin{array}{r}
\nabla\left(\frac{\Pi_{1}}{\rho}\right)-C \nabla \mu-\frac{\phi}{2}\left(\boldsymbol{w} \cdot \boldsymbol{w}_{0}\right) \nabla C=0, \\
\nabla^{2} \mu=0, \mu=2 a_{T} C+4 b C^{3}-\epsilon \nabla^{2} C, \\
\nabla \times \boldsymbol{w}=\phi \boldsymbol{w}_{0} \times \nabla C, \nabla \cdot \boldsymbol{w}=0 .
\end{array}
$$

The solution of the equation for the chemical potential is trivial, $\mu=0$. This solution defines the state of thermodynamic equilibrium for the binary system. The concentration profile for this state is given by the well known tanh-solution,

$$
C(y)=C_{e q} \tanh \left(\frac{y}{\delta_{e q}}\right) .
$$


Here $\delta_{e q}^{2} \equiv-\epsilon / a_{T}$ and $C_{e q}^{2} \equiv-a_{T} /(2 b)$.

Next, we determine the structure of the pulsation field. For this problem it is convenient to introduce the streamfunction, $\psi$, by using the following definitions, $w_{x}=\partial \psi / \partial y$ and $w_{y}=-\partial \psi / \partial x$. We also assume that the imposed vibrational forcing is given by the expression, $\boldsymbol{w}_{0}=a \omega \boldsymbol{j}$, with the direction of the vibrations given by the unit vector $\boldsymbol{j}=\left(j_{x}, j_{y}\right)$. The field of the streamfunction is then determined by the equation

$$
\nabla^{2} \psi=-\phi a \omega\left(j_{y} \frac{\partial C}{\partial x}-j_{x} \frac{\partial C}{\partial y}\right)
$$

For the examined configuration the field of concentration is the sole function of $y$. Hence, the pulsation flow would be absent if the directions of the vibrations is orthogonal to the interface surface. If the vibrations are imposed along the interface, $\boldsymbol{j}=(1,0)$, then there is a no-zero pulsation flow as given below

$$
\boldsymbol{w}=\left(w_{x}, w_{y}\right)=(-\phi a \omega C(y), 0)
$$

Finally, one may also obtain the following expression for the modified pressure field,

$$
\Pi_{1}=\frac{1}{4} \rho(\phi a \omega)^{2} C^{2}(y) .
$$

The obtained expressions define the state of the quasi-equilibrium. The study of the stability of this case would need a much more elaborate analysis due to a long number of the non-dimensional parameters that enter the governing equations. The stability of an interface between two miscible liquids that are just brought into contact and that fill in a container subjected to high-frequency vibrational forcing is the focus of the second part of our work.

\section{REFERENCES}

Ahmadlouydarab, M. \& Feng, J. J. 2014 Motion and coalescence of sessile drops driven by substrate wetting gradient and external flow. J. Fluid Mech. 746, 214-235.

Chella, R. \& Vinals, J. 1996 Mixing of a two-phase fluid by cavity flow. Phys. Rev. E 53, $3832-3840$.

Ding, H., Spelt, P.D.M. \& Shu, C. 2007 Diffuse interface model for incompressible two-phase flows with large density ratios. Journal of Computational Physics 226, 2078-2095.

Gan, L.H, Gan, Y.Y. \& DeEn, G.R. 2000 Poly(n-acryloyl-n-propylpiperazine): a new stimuliresponsive polymer. Macromolecules 33, 7893-7897.

Gaponenko, Y., Torregrosa, M. M., Yasnou, V., Mialdun, A. \& Shevtsova, V. $2015 a$ Interfacial pattern selection in miscible liquids under vibration. Soft Matter. 11, 82218224.

Gaponenko, Y. A. \& Shevtsova, V. 2010 Effects of vibrations on dynamics of miscible liquids. Acta Astronautica. 66, 174-182.

Gaponenko, Y. A., Torregrosa, M., Yasnou, V., Mialdun, A. \& Shevtsova, V. $2015 b$ Dynamics of the interface between miscible liquids subjected to horizontal vibration. J. Fluid Mech. 784, 342-372.

Gaponenko, Y. A., Volpert, V. A., Zen'kovskaya, S. M. \& Pojman, D. A. 2006 Effect of high-frequency vibration on convection in miscible fluids. J. Fluid Mech. 47, 190-198.

Gershuni, G.Z. \& Lyubimov, D.V. 1998 Thermal Vibrational Convection. Wiley \& Sons.

JACQMIN, D. 1999 Calculation of two-phase navierstokes flows using phase-field modeling. Journal of Computational Physics 155, 96-127.

Joseph, D. D. \& Renardy, Y. Y. 1993 Fundamentals of two-fluid dynamics. Part II: lubricated transport, drops and miscible liquids. Springer-Verlag.

Landau, L.D. \& Lifshitz, E.M. 1980 Statistical Physics, 3rd Edition, Part 1. Pergamon Press.

Landau, L.D. \& Lifshitz, E.M. 1987 Fluid Mechanics (Volume 6 of Course of Theoretical Physics). Pergamon Press. 
Legendre, M., Petitjeans, P. \& Kurowski, P. 2003 Instabilités à l'interface entre fluides miscibles par forcage oscillant horizontal. C. R. Mecanique 331 (2), 617-622.

Lowengrub, J. \& Truskinovsky, L. 1998 Quasi-incompressible cahn-hilliard fluids and topological transitions. Proc. R. Soc. London, Ser. A 454 (2), 2617-2654.

Lyubimov, D.V., Lyubimova, T.P. \& Cherepanov, A.A. 2003 Dynamics of interfaces subject to vibrations (in Russian). FizMatLit.

Lyubimov, D., Lyubimova, T., Vorobev, A., Mojtabi, A. \& Zappoli, B. $2006 a$ Thermal vibrational convection in near-critical fluids. part 1. non-uniform heating. J. Fluid Mech. 564, 159-183.

Lyubimov, D., Lyubimova, T., Vorobev, A., Mojtabi, A. \& Zappoli, B. $2006 b$ Thermal vibrational convection in near-critical fluids. part 2 . weakly non-uniform heating. J. Fluid Mech. 564, 185-196.

Lyubimova, T., Vorobev, A. \& Prokopev, S. 2019 Rayleigh-taylor instability of a miscible interface in a confined domain. Physics of Fluids 31, 014104.

Mason, Warren P., ed. 1965 Physical Acoustics, Volume II Part B: Principles and Methods, Properties of Polymers and Nonlinear Acoustics. Academic Press.

Pojman, J. A., Whitmore, C., Liveri, M. L. T., Lombardo, R., Marszalek, J., Parker, R. \& Zoltowski, B. 2006 Evidence for the existence of an effective interfacial tension between miscible fluids: isobutyric acid-water and 1-butanol-water in a spinning-drop tensiometer. Langmuir 22, 2569-2577.

Prokopev, S, Vorobev, A. \& Lyubimova, T. 2019 Phase-field modeling of an immiscible liquid-liquid displacement in a capillary. Phys. Rev. E 99, 033113.

Schlichting, H. \& Gersten, K. 2017 Boundary-layer theory. Springer-Verlag Berlin Heidelberg.

Shevtsova, V., Gaponenko, Y., Yasnou, V., Mialdun, A. \& Nepomnyashchy, A. 2015 Wall-generated pattern on a periodically excited miscible liquid/liquid interface. Langmuir. 31, 5550-5553.

Shevtsova, V., Gaponenko, Y. A., Yasnou, V., Mialdun, A. \& Nepomnyashchy, A. 2016 Two-scale wave patterns on a periodically excited miscible liquid-liquid interface. J. Fluid Mech. 795, 409-422.

Umantsev, A. 2002 Thermal effects in dynamics of interfaces. Journal of Chemical Physics 116, 4252-4265.

Vorobev, A. 2010 Boussinesq approximation of the cahn-hilliard-navier-stokes equations. Phys. Rev. E 82 (10), 056312.

Vorobev, A. 2014 Dissolution dynamics of miscible liquid/liquid interfaces. Current Opinion in Colloid $\mathcal{G}$ Interface Science 19, 300-308.

Vorobev, A. \& Boghi, A. 2016 Phase-field modelling of a miscible system in spinning droplet tensiometer. Journal of Colloid and Interface Science 482, 193-204.

Vorobev, A., Ivantsov, A. \& Lyubimova, T. 2017 Phase-field modelling of gravity-capillary waves on a miscible interface. Eur. Phys. J. E 40, 99.

Vorobev, A. \& Khlebnikova, E. 2018 Modelling of the rise and adsorption of a fluid inclusion. Int. J. Heat Mass Trans. 125, 801-814.

Wolf, G. H. 2018 Dynamic stabilization of the rayleigh-taylor instability of miscible liquids and the related "frozen waves". Physics of Fluids. 30, 021701.

XIE, R. \& Vorobev, A. 2016 On the phase-field modelling of a miscible liquid/liquid boundary. Journal of Colloid and Interface Science 464, 48-58.

Zappoli, B., Beysens, D. \& Garrabos, Y. 2015 Heat Transfers and Related Effects in Supercritical Fluids. Springer. 